\title{
Ferrostatin-1 Alleviates White Matter Injury via Decreasing Ferroptosis Following Spinal Cord Injury
}

\section{Hongfei Ge}

Third Military Medical University: Army Medical University

Xingsen Xue

Third Military Medical University: Army Medical University

Jishu Xian

Third Military Medical University: Army Medical University

Linbo Yuan

Third Military Medical University: Army Medical University

\section{Long Wang}

Third Military Medical University: Army Medical University

\section{Yongjie Zou}

Third Military Medical University: Army Medical University

Jun Zhong

Third Military Medical University: Army Medical University

\section{Zhouyang Jiang}

Third Military Medical University: Army Medical University

\section{Jiantao Shi}

Third Military Medical University: Army Medical University

\section{Tunan Chen}

Third Military Medical University: Army Medical University

\section{Hong Su}

Third Military Medical University: Army Medical University

\section{Hua Feng}

Third Military Medical University: Army Medical University

Shengli Hu ( $\sim$ husl1937@163.com )

Third Military Medical University: Army Medical University https://orcid.org/0000-0001-6438-6924

\section{Research Article}

Keywords: spinal cord injury, ferroptosis, white matter injury, reactive oxygen species, ferrostatin-1

Posted Date: April 15th, 2021

DOI: https://doi.org/10.21203/rs.3.rs-405886/v1

License: (c) (i) This work is licensed under a Creative Commons Attribution 4.0 International License. Read Full License

Version of Record: A version of this preprint was published at Molecular Neurobiology on October 12th, 2021. See the published version at https://doi.org/10.1007/s12035-021-02571-y. 


\section{Abstract}

Spinal cord injury (SCl), a devastating neurological impairment, ubiquitously imposes a long-term psychological stress and high socioeconomic burden for the suffers and their family. To date, recent researchers have paid arousing attention to white matter injury and uncovering the underlying mechanism post-SCl. Ferroptosis, to our knowledge, has been revealed to be associated with diverse diseases including stroke, cancer, and kidney degeneration. However, its role in white matter damage after $\mathrm{SCl}$ remains unclear. Ferrostatin-1, a potent inhibitor of ferroptosis, has been illustrated to curb ferroptosis in neurons, subsequently improve functional recovery after traumatic brain injury (TBI). But whether it inhibits white matter injury post-SCl is still unknown. Here, our results indicated that ferroptosis played an important role in the secondary white matter injury following $\mathrm{SCl}$ and ferrostatin-1 could reduce iron and reactive oxygen species (ROS) accumulation, downregulate the ferroptosis-related genes and its products of IREB2 and PTGS2 to further inhibit ferroptosis in oligodendrocyte progenitor cells (OPCs), finally reducing white matter injury and promoting functional recovery following $\mathrm{SCl}$ in rats, which enlarges the therapeutic scope for ferrostatin- 1 and deciphers the potential mechanism of white matter damage after SCl.

\section{Introduction}

Spinal cord injury (SCI), a devastating neurological impairment, usually occurs in subpopulation with workforce, and imposes a long-term psychological stress and high socioeconomic burden for the suffers, as well as their family [1-3]. Hence, exploring novel strategies for SCl has sparked great interest for researchers. Our past studies have uncovered a variety of therapeutic candidates associated with secondary injury, including promoting neuron survival through inhibiting acid-sensing ion channel 1a (ASIC 1a) [4] or activating G-protein coupled estrogen receptor 1 (GPER1) [2,5], mediating neuroinflammation by complement C5a [6], decreasing glial scar formation and potentiating axon regeneration using curcumin, antisense vimentin CDNA combined with chondroitinase ABC [7-9], and cell-based strategies using exogenous transplantation of human umbilical cord mesenchymal stem cells [10] and directing endogenous neural stem cells (NSCs) differentiation into neurons [11,12]. However, majority of SCl patients still recover poorly in clinic, implying that more mechanisms need to be elucidated with respect to secondary injury, and exploring more effective treatment for $\mathrm{SCl}$ is of great significance.

The main focus of investigators is to devise therapeutic strategies in order to promote neuronal regeneration following SCl during the last four decades. While, studies have revealed that $5-10 \%$ of the original number of axons survives during the acute phase of SCl, which would be lost during the chronic stage without appropriate intervention [13-15]. Furthermore, evidence have shown that the limited number of epibiotic axons holds sufficient potential in facilitating locomotor function recovery post-SCI [13-15], suggesting that promoting remyelination during the acute phase might be a feasible strategy for $\mathrm{SCl}$ treatment. Oligodendrocytes are a subtype of neural cells, which are mainly responsible for myelination in the central nervous system (CNS) $[13,16]$. And, previous studies have indicated that oligodendrocytes are susceptible to oxidative damage due to high level of iron deposition induced by hemorrhage in the epicenter of the spinal cord after SCI [17-19]. Herein, immediately reducing the concentration of iron during the acute phase of $\mathrm{SCl}$ is supposed to preserve oligodendrocytes, thereafter, facilitating functional recovery. Coincidently, our recent research has presented that using deferoxamine (DFX), one of iron chelators, holds the ability of reducing axonal damage and demyelination through downregulation of transferrin receptor and divalent metal transporter 1 (DMT1) to decrease iron overload [20]. Additionally, ferroptosis, a novel form of regulated cell death (RCD) [21], must be triggered due to high level of iron accumulation. Most recently, investigation also presents that Liproxstatin-1 (Lipro-1), an inhibitor of ferroptosis, protects oligodendrocytes from ferroptosis through inhibiting lipid peroxidation and increasing expression of glutathione (GSH), glutathione peroxidase 4 (GPX4), and ferroptosis suppressor protein 1 (FSP1) [19].

Ferrostatin-1, another potent inhibitor of ferroptosis, is synthetically isolated by high-throughput screening of small molecule libraries [22]. Recent studies have illustrated that ferrostatin-1 bears the ability of scavenging hydroperoxyl radical to reduce cell death through mitigating ferroptosis $[23,22]$. Additionally, another study has found that ferrostatin-1 bears the potential of suppressing neuroinflammation and ferroptosis resulted from

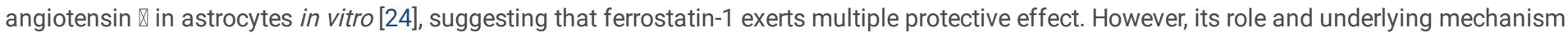
after spinal cord injury is still indefinable.

In the present study, we hypothesized that oligodendrocytes ferroptosis induced by iron overload introduced an important role in the secondary white matter injury following $\mathrm{SCl}$, and administration of ferrostatin-1 could reduce oligodendrocytes ferroptosis via reducing reactive oxygen species (ROS) production, thereafter attenuating white matter injury to promote functional recovery post-SCl in rats. The aim of the present study is to certify the effect of ferrostatin-1 on $\mathrm{SCl}$ in rats, and to offer a possible therapeutic strategy for $\mathrm{SCl}$ treatment from bench to bedside.

\section{Materials And Methods}

\section{Animals}

All experiments were conducted in accordance with the China's animal welfare legislation for the protection of animals used for scientific purposes. And all procedures were supervised by the Ethics Committee of the Southwest Hospital, Third Military Medical University for the use of laboratory animals (approval no. SYXK 20170002). A total of 126 adult female (200-250 g, 118 rats used for experiments and 8 rats died during experiments) 
Wistar rats and 50 pregnant Wistar rats were used in the present research. All rats were given free access to food and water under the condition of constant photoperiod (12-h light/dark cycle), temperature $\left(22-25^{\circ} \mathrm{C}\right)$ and moisture $(55-60 \%)$ before and after surgery. Every effort was made to reduce the number, and to alleviate their sufferings of rats.

\section{Surgical procedures}

The surgical procedures were carried out under sterile condition as previously described [20,5,2] . In brief, rats were placed in a stereotaxic frame after anesthesia with $2 \%$ isoflurane/air mixture (2-3 l/min). A 4-cm-long incision was made in the skin along the midline of the back, and a laminectomy was carried out to expose the thoracic 9-11 spinal segments, leaving the dura intact. Thereafter, spinal contusion injury was performed using a 20-g weight rod (diameter $4 \mathrm{~mm}$ ) dropping from a height of $30 \mathrm{~mm}$ onto the exposed T10 rat spinal cord. Afterward, the muscles and skin were sutured in separate layers. Body temperatures were maintained at $37 \pm 0.3^{\circ} \mathrm{C}$ on a heating pad throughout the procedure. Rats were received manual bladder empty twice a day until they could do themselves, and their weight loss, dehydration, autophagia and discomfort were recorded each day, even with appropriate veterinary care if needed.

\section{Experimental groups}

After surgery, rats were randomly assigned into the following groups:

i) Sham group. Rats $(n=10)$ were received laminectomy without contusion. Then rats were intra-spinal cord microinjected with the same volume of $9 \% \mathrm{NaCl}$ (containing $0.1 \% \mathrm{DMSO}$ ), equivalently to the volume of ferrostatin-1 in SCl+ferrostatin-1 group, using the same method.

ii) SCl group. Rats $(n=15)$ were received laminectomy with contusion. Then rats were intra-spinal cord microinjected with the same volume of $9 \%$ $\mathrm{NaCl}$ (containing $0.1 \% \mathrm{DMSO}$ ), equivalently to the volume of ferrostatin-1 in SCl+ferrostatin-1 group.

iii) SCl + ferrostatin-1 group. Rats ( $\mathrm{n}=15)$ were received laminectomy with contusion. Four hours after surgery, ferrostatin-1 (0.7 mg/kg, SigmaAldrich, Munich, Germany), which was diluted in $0.9 \% \mathrm{NaCl}$ after dissolved in DMSO, was microinjected into the dorsal spinal cord 2 mm rostrally and $2 \mathrm{~mm}$ caudally to the injury site at a depth of $1.2 \mathrm{~mm}$ and $0.75 \mathrm{~mm}$ laterally from midline at a rate of $1 \mu \mathrm{l} / \mathrm{min}$. The needle was left in position for a further $2 \mathrm{~min}$ before being slowly withdrawn. A total volume of $10 \mu$ Ferrostatin- 1 was injected. The second treatment was on the second day after SCl.

\section{Behavioral test}

The locomotor recovery of rats in different groups was evaluated using the Basso, Beattie, and Bresnahan (BBB) locomotion score by two independent examiners blinded to the experimental groups on days $3,7,21,35$, and 56, as previously described [25,3]. The score ranges from 0 to 21 , where 0 reflects no locomotor function and 21 reflects normal performance. Rats were allowed to walk around freely in a 90 -cm ${ }^{2}$ field for 5 mins, while movements of the hind limb were closely observed.

\section{Primary oligodendrocyte progenitor cells (OPCs) culture}

Primary OPCs were isolated from P0-P1 Wistar rats as previously described [26]. Cells were cultured in Dulbecco's modified Eagle's medium (DMEM; Hyclone, Logan, Utah, USA) supplemented with 20\% fetal calf serum. After 10-12 days, immature oligodendrocytes, attached to the astrocyte layer of the mixed glia culture, were isolated by shaking overnight at $230 \mathrm{rpm}$. The collected cells were pre-seeded for 30 min at $5 \% \mathrm{CO}_{2}$ and $37^{\circ} \mathrm{C}$ to remove contaminating microglia and astrocytes. Floating cells were collected in DMEM supplemented with B27 (Gibco, Grand Island, NY, USA), 10 ng/ml plated derived growth factor (PDGF, Peprotech, Rocky Hill, NJ, USA), 10 ng/ml basic fibroblast growth factor (bFGF, Peprotech, Rocky Hill, NJ, USA) and plated on poly-L-ornithine-coated (Sigma-Aldrich, Munich, Germany) cover slips or culture plates ( $1 \times 10^{5}$ cells/ml). At this stage the purity of culture was approximately around $95 \%$, assessed by expression of the immature oligodendrocyte surface progenitor markers of Olig2 and 04. Ferrostatin-1 was first dissolved in DMSO and then diluted with culture medium (containing $0.1 \%$ DMSO) with the final concentration of $0.5 \mu \mathrm{M}$ for in vitro experiments. The control or vehicle group was added the same volume of DMSO as the ferrostatin-1 group.

\section{Perl's Prussian blue staining}

Rats $(n=5)$ were transcardiacally perfused with $0.1 \mathrm{M}$ phosphate buffered saline (PBS), followed by $4 \%$ paraformaldehyde (PFA, pH 7.2-7.4) in 0.1 M PBS after anesthesia with $2 \%$ isoflurane/air mixture $(2-3 \mathrm{l} / \mathrm{min})$ on day 7 . The T10 spinal cord segment containing the injury epicenter and surrounding uninjured tissues ( $6 \mathrm{~mm}=3 \mathrm{~mm}$ either side from the injury epicenter) were dissected and prepared for paraffin sections according to the standard procedures. Iron accumulation in spinal cords or primary OPCs was determined using the Perl's Prussian blue staining method, as previously described $[20,27]$. Paraffin sections $(5 \mu \mathrm{m})$ from spinal cords were processed through a graded ethanol series, immersed in xylene, and then rehydrated in PBS. Subsequently, samples were immersed in Perls' Staining Solution (comprising equal parts of potassium ferrocyanide and $\mathrm{HCL}$ ) for 20 min. Afterward, the samples were washed with Milli-Q water and stained with nuclear fast Red for 5-10 min, dehydrated, cleared in xylem and mounted using a standard procedure. Deposits of iron were stained as blue by Perl's Prussian blue staining, while cytoplasm and cellular nucleus were stained as pink and red, respectively. 
Spinal cord samples $(n=8)$ or primary OPCs were collected and homogenized under ice-cold conditions. Iron levels were measured using the tissue iron assay kit (Nanjing Jiancheng Bioengineering Institute, Nanjing, China) in accordance with manufacturer's instruction. To determine iron concentration, the optical density (OD) value was measured at $520 \mathrm{~nm}$ using a spectrophotometer (Varioskan Flash, Thermo Scientific, Waltham, MA, USA) and the iron concentration in all samples was then determined through comparing the OD of the samples to the standard curve.

\section{Reactive oxygen species (ROS) measurement}

ROS levels were determined by a reactive oxygen species assay kit according to the manufacturer's specification (cat. no. S0033S, Beyotime Biotechnology, Beijing, China). Spinal cord samples $(n=3)$ or primary OPCs were collected and homogenized under ice-cold conditions. Each sample was loaded $500 \mu \mathrm{l}$ of $10 \mu \mathrm{M}$ DCFH-DA and incubated in the dark at $37^{\circ} \mathrm{C}$ for $20 \mathrm{~min}$. The loading buffer was replaced and washed three times to eliminate residual DCFH-DA. The samples were then immediately measured using a Flow cytometer (ACEA Biosciences Inc., San Diego, CA, USA) with an argon laser $(488 \mathrm{~nm})$.

\section{Immunohistochemistry (IHC)}

For immunofluorescence staining, slices $(n=9)$ were incubated in $4 \%$ paraformaldehyde in $0.01 \mathrm{M}$ PBS for 30 min at room temperature and permeabilized with $0.3 \%$ Triton-X 100 (Sigma-Aldrich, St. Louis, MO) in PBS. Then, samples were incubated in the following antibodies overnight at $4^{\circ} \mathrm{C}$ after blocked with $5 \%$ bovine serum album (BSA, Sigma-Aldrich, St. Louis, MO): anti-APP antibody (cat. no. MAB348, Sigma-Aldrich, Munich, Germany), anti-dMBP (cat. no. MBS618031, MybioSource, San Diego, CA, USA), anti-Olig2 (cat. no. ab109186, Abcam, Cambridge, UK), or anti-04 (cat. no. MAB345, Sigma-Aldrich, Munich, Germany). Samples were then incubated in relative secondary antibodies for 2 hours at room temperature. The cell nuclei were counterstained with 4'-6-Diamidino-2-phenylindole (DAPI, Beyotime, Beijing, China) for 10 minutes at room temperature. Samples were mounted onto glass slides, and images were captured using a confocal microscope (Carl Zeiss, LSM780, Weimar, Germany) and examined by Zen 2011 software (Carl Zeiss, Weimar, Germany).

For immunohistochemistry, sections were dewaxed and antigen-repaired according to the standard procedures [28]. Next, slices were incubated in endogenous peroxidase for $10 \mathrm{~min}$. Samples were incubated in anti-CC1 (cat. no. SAB4501438, Sigma-Aldrich, Munich, Germany) overnight at $4^{\circ} \mathrm{C}$ after blocked with 5\% BSA dissolved in 0.5\% v/v Triton-X 100 (Sigma-Aldrich, St. Louis, MO) in PBS. After washing, they were incubated in horseradish peroxidase (HRP)-conjugated goat anti-mouse immunoglobulin G (ZSGB-BIO, Beijing, China). Then, the 3-diaminobenzidine (DAB) kit was employed to stain in color. Sections were counterstained with hematoxylin and dehydrated with ethanol and xylene to prep for mounting. Thereafter, coverslips were mounted onto glass slides. Images were captured using a light microscope (Olympus, Tokyo, Japan).

For each sample, six sections were immunostained, analyzed, and the cross-sectional areas were calculated and reported as the average of four independent measurements. All measurements were performed by an individual investigator who was blinded to the experiment groups.

\section{Western blot}

The T10 spinal cord segments $(n=3)$ containing the injury epicenter and surrounding uninjured tissues $(0.5 \mathrm{~cm}=0.25 \mathrm{~cm}$ either side from the injury epicenter) were immediately dissected after decapitation on day 7 post-SCI. The tissue lysates were collected, and the protein concentration of each sample was determined using a Bicinchoninic Acid (BCA) method (Beyotime, Beijing, China). Proteins ( $50 \mu \mathrm{g})$ were separated by $10 \%$ SDS-PAGE under reducing conditions and electro-blotted to polyvinylidene difluoride (PVDF, Roche, Indianapolia, IN, USA) membranes. Then, the membranes were incubated in 5\% (w/v) non-fat dry milk (Beyotime Institute of Biotechnology) in TBS with Tween-20 (TBST) at room temperature for $2 \mathrm{~h}$. Then, the membranes were cut out at different parts according to a pre-stained protein molecular ladder (cat. no. 26616; Thermo Fisher Scientific, Inc., Waltham, MA, USA) to allow separate detection of proteins migrating at the same distance, and were incubated in primary antibodies, anti-APP antibody (cat. no. MAB348, Sigma-Aldrich, Munich, Germany), anti-dMBP (cat. no. MBS618031, MybioSource, San Diego, CA, USA), anti-IREB2 antibody (cat. no. SAB2501280, Sigma-Aldrich, Munich, Germany), anti-PTGS2 (cat. no. ab179800, Abcam, Cambridge, UK), or anti-GAPDH (cat. no. AF0006; Beyotime Institute of Biotechnology) overnight at $4^{\circ} \mathrm{C}$. Afterward, the membrane was incubated in corresponding horseradish peroxidase

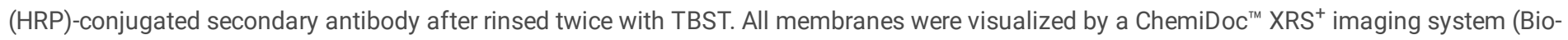
Rad, California, USA) using the WesternBright ECL Kits (Advansta, Menlo Park, CA, USA). Densitometric measurement of each membrane was performed using Image Lab ${ }^{T M}$ software (Bio-Rad, California, USA).

\section{Reverse Transcription-quantitative Polymerase Chain Reaction (RT-qPCR)}

Total RNA was extracted using TaKaRa MiniBEST Universal RNA Extraction Kit (TaKaRa, Tokyo, Japan) according to the manufacturer's instructions after primary OPCs were collected from each group. Thereafter, $1 \mu \mathrm{g}$ RNA was reverse transcribed into cDNA using PrimeScript RT reagent Kit with gDNA Eraser (cat. no. RR0047A, TaKaRa, Tokyo, Japan) according to the manufacturer's direction. Subsequently, qPCR was performed using CFX96 System (Bio-Rad, CA, USA) with SYBR Premix Ex Taqll (Tli RNaseH Plus) (cat. no. RR820A, TaKaRa, Tokyo, Japan) under the following condition: $95^{\circ} \mathrm{C}$ for $30 \mathrm{sec}, 40$ cycles at $95^{\circ} \mathrm{C}$ for $5 \mathrm{sec}$ and $60^{\circ} \mathrm{C}$ for $30 \mathrm{sec}$. Relative mRNA levels were normalized to GAPDH and analyzed using the $2-\Delta \Delta \mathrm{Cq}$ method. Primer sequences used in the present study were as follows:

Page 4/14 


\begin{tabular}{|c|c|c|}
\hline Primer & Forward & Reverse \\
\hline IREB2 & 5'-GAGACTGGGCTGCGAAAGGA-3' & $\begin{array}{l}5^{\prime}- \\
\text { CCTGGGAGGAACTCAAGTGGTG-3' }\end{array}$ \\
\hline PTGS2 & 5'-TCACCCGAGGACTGGGCCAT-3' & $\begin{array}{l}\text { 5'- } \\
\text { TGGGAGGATACACCTCTCCACCG- } \\
3^{\prime}\end{array}$ \\
\hline GAPDH & $\begin{array}{l}\text { 5'- } \\
\text { AACCTGCCAAGTATGATGACATCA- } \\
3^{\prime}\end{array}$ & $\begin{array}{l}5^{\prime}- \\
\text { TGTTGAAGTCACAGGAGACAACCT- } \\
3^{\prime}\end{array}$ \\
\hline
\end{tabular}

\section{Transmission electron microscopy (TEM)}

TEM was performed to visualized ultrastructural of primary OPCs in different groups, as previously described [28]. Primary OPCs from each group were incubated in $2 \%$ glutaraldehyde overnight at $4{ }^{\circ} \mathrm{C}$ and then transferred to $1 \%$ citric acid for fixation. Thereafter, they were dehydrated with gradient acetone after soaking in uranyl acetate. Subsequently, samples were embedded with epoxy resin and sliced into $70-90$ nm. Then, they were counterstained with lead citrate after placing on the copper trough grid and the ultrastructure of mitochondria was observed using a transmission electron microscope (Hitachi HT7700, Chiyoda-ku, Tokyo, Japan). At least three independent samples per group were used for TEM analysis.

\section{Statistical analysis}

Statistical analysis was performed using Graphpad version 9.0 (Graphpad Software Inc., San Diego, CA, USA). Comparisons between two groups were analyzed using analysis of variance (ANOVA), followed by Tukey's post hoc test in case of the data were normality using a Shapiro-Wilk normality test. Meanwhile, data failing the normality test were represented as median and interquartile range (IQR) using the Mann-Whitney $U$ test. A $p<0.05$ was considered as significant difference.

\section{Results}

Ferrostatin-1 improved motor behavior through decreasing ROS accumulation in rats after SCl.

To evaluate the effect of ferrostatin-1 on functional recovery after SCI, the BBB score was firstly performed. The results indicated that the BBB score of rats in $\mathrm{SCl}+$ ferrostatin-1 group was evidently higher than that in $\mathrm{SCl}$ group from day 7 to 56 (Figure 1A). Given that ferrostatin-1 is an inhibitor of ferroptosis and iron is the main trigger of ferroptosis, we then determined the iron concentration. The results showed that ferrostatin- 1 significantly reduced the iron concentration, whose level was greatly elevated in spinal cords after SCI (Figure 1B). Next, the Perl's Prussian blue staining was conducted to verify the iron deposition in the epicenter of injured spinal cords. The images delineated that ferrostatin-1 dramatically reduced the iron accumulation in the epicenter of injured spinal cords (Figure 1C). With respect to the high level of ion could directly or indirectly upregulate the ROS accumulation after injury [29], we thereafter determined the level of ROS in the epicenter of damaged spinal cords. The results indicated that ferrostatin-1 predominantly reduced the ROS fluorescence, which was substantially increased in the injured spinal cords, on days 3,7 and 21 (Figure 2A, B). Collectively, these results illustrated that ferrostatin-1 facilitated locomoter deficits via deducing iron and ROS accumulation post-SCI in rats.

\section{Ferrostatin-1 attenuated white matter injury after $\mathrm{SCl}$ in rats.}

Given that oligodendrocytes are susceptible to oxidative damage and high level of iron deposition after SCI [17-19], the average number of oligodendrocytes was firstly evaluated using immunochemistry. The results depicted that the number of $\mathrm{CC} 1^{+}$, which is usually used to specifically label mature oligodendrocytes [30], was dramatically deduced in the epicenter of spinal cords after SCl, while ferrostatin-1 partially abrogated this effect, that was administration of ferrostatin reduced oligodendrocytes loss (Figure 3A, B). Next, the expression of degraded myelin basic protein (dMBP), a symbol of myelin degradation, and APP-a maker of damaged axons, was determined using western blot assays. The bands depicted that 
the expression of dMBP and APP was substantially elevated in the surrounding epicenter of spinal cord after SCl in rats (Figure 3C-E). However, the enhanced expression of dMBP and APP was obviously downregulated with ferrostatin- 1 treatment (Figure 3C-E). Moreover, the immunostaining images represented that ferrostatin-1 evidently decreased the dMBP intensity that was upregulated resulting from SCI (Figure 3F, G). Subsequently, the immunostaining of APP showed the similar phenomenon as AMBP, and further attested the results obtained from western blot assays (Figure $3 \mathrm{H}, \mathrm{I})$. Mechanically, these results suggested that ferrostatin- 1 held the ability of attenuating demyelination via decreasing the oligodendrocytes loss after SCl.

\section{Ferrostatin-1 facilitated oligodendrocyte progenitor cells (OPCs) survival through decreasing ferroptosis in vitro.}

To further unravel the underlying mechanism that ferrostatin-1 enhanced oligodendrocytes survival in vivo, the primary OPCs were isolated from P0-P1 Wistar rats. As shown in Figure 4A, most of the cultured cells expressed Olig2 and 04, and exhibited the morphology of OPCs. Considering

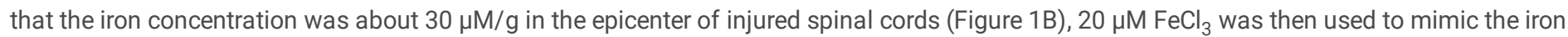
concentration in ex-vivo experiments. The results showed that $20 \mu \mathrm{M} \mathrm{FeCl}_{3}$ greatly reduced OPCs viability from $48 \mathrm{~h}$ to $72 \mathrm{~h}$, and this deduced viability could be partially reversed with addition of $0.5 \mu \mathrm{M}$ ferrostatin-1 (Figure 4B). Thereafter, the iron deposition in primary OPCs was evaluated

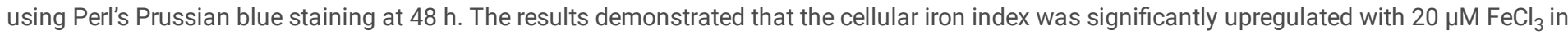
groups $\mathrm{FeCl}_{3}$ and $\mathrm{FeCl}_{3}+$ vehicle (Figure 4C, D). However, this effect was prevalently abolished with addition of $0.5 \mu \mathrm{M}$ ferrostatin- 1 , in a degree (Figure 4C, D). In addition, the TEM was conducted to observe the morphology of mitochondria and the results showed that the proportion of shrunken mitochondria was markedly increased in groups of $\mathrm{FeCl}_{3}$ and $\mathrm{FeCl}_{3}+$ vehicle (Figure $5 \mathrm{E}, \mathrm{F}$ ), while $0.5 \mu \mathrm{M}$ ferrostatin-1 abolished this phenomenon, to some extent (Figure 5E, F). In sum, these results illustrated that ferrostatin-1 bore the potentiality of reducing iron accumulation and ROS deposition in OPCs to decrease iron-overload damage in vitro.

\section{Ferrostatin-1 mitigated OPCs ferroptosis resulting from iron overload through reducing IREB2 expression.}

With respect to ferrostatin-1 potentiates hydroperoxyl radical scavenging to reduce ferroptosis [23,22], and reduced iron-overload in neurons after traumatic brain injury (TBI) [31], we then tested the effect of ferrostatin-1 on OPCs ferroptosis in vitro. First, the RT-qPCR assays indicated that the expression of IREB2 and PTGS2 mRNA was significantly increased from $24 \mathrm{~h}$ to $72 \mathrm{~h}$ (Figure $5 \mathrm{~A}$ ). Then, ferrostatin- 1 was used to investigate its role in the expression of IREB2 and PTGS2 mRNA. The results represented that ferrostatin-1 partially decreased the expression of IREB2 and PTGS2 mRNA, which was predominantly elevated with $\mathrm{FeCl}_{3}$ addition at $48 \mathrm{~h}$ (Figure $5 \mathrm{C}, \mathrm{D}$ ). Thereafter, the western blot assays were performed to certify the results obtained from RT-qPCR. The results indicated that the expression of IREB2 and PTGS2 was dramatically increased with FeCl 3 addition from $24 \mathrm{~h}$ to $72 \mathrm{~h}$, and both of IREB2 and PTGS2 reached the peak at $48 \mathrm{~h}$ (Figure 5E-G). Subsequently, ferrostatin- 1 was added to determine its role in attenuating ferroptosis induced by $\mathrm{FeCl}_{3}$. The results displayed that ferrostatin-1 deduced the expression of IREB2 and PTGS2 at $48 \mathrm{~h}$ (Figure $5 \mathrm{H}$, I). Mechanically, these results represented that iron accumulation resulted in upregulation of ferroptosis-related genes and proteins, as well as increase of shrunken mitochondria, while ferrostatin-1 eliminated this effect, to some extent.

\section{Discussion}

The present study provides evidence that iron accumulation occurs after spinal cord injury, which may lead to severe white matter injury and locomotor function deficits. Iron overload induces ROS production, and then oligodendrocytes ferroptosis. Moreover, ferrostatin-1, one specific inhibitor of ferroptosis, reduces the content of ROS, alleviates oligodendrocytes ferroptosis, attenuates white matter damage, and promotes neurological functional recovery in rats subjected to $\mathrm{SCl}$. These data clearly indicate that ferroptosis elicited by iron overload plays an important role in the secondary white matter injury post-SCI. Inhibition of ferroptosis with ferrostatin-1 may contribute to neurological outcome recovery of patients obsessed by SCl.

It is generally believed that white matter injury serves as a potential role in contributing neurological loss after SCl. In recent years, more and more attention has been paid to white matter repair after $\mathrm{SCl}$, which is considered as a key factor for functional recovery post-SCl. Obtained evidence shows that white matter damage induced by SCl not only occurs in a wide area around the damaged site, but also extends beyond a few millimeters [13]. Oligodendrocytes loss usually peaks at 7 days, and persists for up to 2 weeks after injury [32,33]. Our previous study also demonstrated severe white matter injury after $\mathrm{SCl}$, which is further confirmed by the present study [20]. Although, white matter injury is a common belief in $\mathrm{SCl}$, the underlying precise mechanism is still unclear and deserves further study. In our recent study, we found that there was remarkable iron deposition in spinal cord tissue after $\mathrm{SCl}$, which activated the iron transport system, enhanced the expression of DMT1 and transferrin receptor (TfR) at protein level and then resulted in intracellular iron overload, accompanied by white matter injury [20]. However, deferoxamine (DFX), one of the iron chelators, could reduce iron accumulation and lessen white matter damage, which indicates that iron overload contributes to white matter injury post-SCI [20]. Then, this presents a new problem: how does iron accumulation trigger the prolonged white matter injury and oligodendrocytes loss?

Ferroptosis, which has been recognized as a new form of cell death [21], has been acknowledged to be intrinsically linked to iron. Therefore, we detect whether ferroptosis correlate closely with white matter injury after SCl. The results showed that ferrostatin-1, a specific inhibitor of ferroptosis, notably reduced white matter injury following $\mathrm{SCl}$, which indicates that ferroptosis possesses an essential role in the secondary white

Page 6/14 
matter injury post-SCI. To further determine whether iron induced oligodendrocytes ferroptosis, we established an in vitro model using primary OPCs treated with $\mathrm{FeCl}_{3}$. Our results suggested that iron could lead to the ferroptosis of cultured OPCs, while ferrostatin-1 ameliorated OPCs ferroptosis caused by $\mathrm{FeCl}_{3}$. Taken together, this study suggests that ferroptosis induced by iron overload plays a key role in the white matter injury following $\mathrm{SCl}$.

Ferroptosis, to our knowledge, has been revealed to be associated with diverse diseases including stroke (intracerebral hemorrhage and ischemia stroke), cold damage, TBI, cancer, and kidney degeneration [31,21,34-40]. In the present study, our results provided a convincing evidence that ferroptosis played an important role in oligodendrocytes damage after SCl. Oligodendrocytes are the main neural lineage cells, which are responsible for myelination in the CNS, while demyelination, which usually retards rapid nerve conduction, is a common phenomenon after SCI. Furthermore, previous studies have represented that reinforcing remyelination during the acute phase of $\mathrm{SCl}$ is a beneficial strategy to protect denuded axons from degeneration and to maintain neurological functions that would be lost otherwise during the chronic stage [13]. Thus, our study offers a new insight into the mechanism of white matter injury post-SCl.

Additionally, past reports have illustrated that IREB2 is a sensitive indicator of ferroptosis, whose upregulation is associated with elevated ferroptosis in pathological process and anti-tumor growth [41-43], which is line with our findings in the present study. Besides, our results demonstrated that ferrostatin-1 could also decrease iron deposition post-SCl, which is in consistent with a previous study on traumatic brain injury (TBI) [31]. Ferroptosis is considered as an iron- and ROS-dependent form of regulated cell death whose induction depends on the production of ROS. The present results demonstrate ferrostatin-1 simultaneously reduces iron and ROS deposition to trigger neuroprotective effect by inhibition of ferroptosis after SCI.

Iron-overload not only induces ferroptosis, but also results in ROS surplus, and vice versa [44]. This positive loop exaggerates neural lineage cells loss after central nervous system (CNS) injury. Hence, reducing iron-overload is a central link to interrupt this vicious paradigm, and provides a therapeutic target for CNS injury. Here, our results proved that the administration of ferrostatin-1 reduced iron and ROS deposition and then white matter ferroptosis in vitro and in vivo. Meanwhile, another study has found that ferrostatin-1 protects neuronal loss through downregulation of ferroptosis after TBI [31]. Moreover, ferrostatin-1 might also facilitate astrocytes preservation through blocking angiotensin $\otimes$ to alleviate neuroinflammation and ferroptosis [24]. Overall, ferroptosis is a central link between various pathological processes, and ferrostatin-1 could interrupt this vicious circle to rehabilitate local neuro-network after SCl. These results enlarge the scope of ferrostatin-1 in treating CNS injury.

In addition, a recent research showed that liproxstatin-1, one of the ferroptosis inhibitor, suppresses ferroptosis through attenuating mitochondrial lipid peroxidation, and upregulating the expression of glutathione (GSH), glutathione peroxidase 4 (GPX4) and ferroptosis suppressor protein 1 (FSP1) in vitro [19]. We further tested the role of ferrostatin-1, another ferroptosis inhibitor, in protecting white matter from ferroptosis in vivo and in vitro. However, the regulated mechanism of ferroptosis post-SCl is not clear and remains to be elucidated in our future work.

In conclusion, this study reveals the novel findings that iron accumulation causes ROS production and then oligodendrocytes ferroptosis, which may produce a central role in the secondary white matter injury after SCl. Administration of ferrostatin-1 may be a feasible therapeutic strategy and beneficial for $\mathrm{SCl}$ patients and their family. Although functional recovery is significantly promoted in the ferrostatin-1 treated animals compared with control group, the direct oligodendrocytes ferroptosis is not identified in vivo. Therefore, further research is needed to verify oligodendrocytes ferroptosis by using immuno-electron microscope method. Subsequently, we still need to elucidate the exact regulatory mechanism of oligodendrocytes ferroptosis after SCl.

\section{Declarations}

\section{Funding}

This work was supported by grants from the National Natural Science Foundation of China (approval no. 81471261) and Natural Science Foundation of Chongqing (approval no. cstc2018jcyjAX0080).

\section{Compliance with Ethical Standards}

\section{Disclosure of potential conflicts of interest}

The authors have no relevant financial or non-financial interests to disclose.

\section{Research involving Animals}

All experiments were conducted in accordance with the China's animal welfare legislation for the protection of animals used for scientific purposes. And all procedures were supervised by the Ethics Committee of the Southwest Hospital, Third Military Medical University for the use of laboratory animals (approval no. SYXK 20170002).

\section{Informed consent}


Not applicable.

\section{Consent to participate}

Not applicable.

\section{Consent for Publication}

Not applicable.

\section{Author contributions}

H.F.G. and X.S.X. performed most of the experiments, with assistance from J.S.X., L.B.Y., Y.J.Z., J.Z., J.T.S., L.W., Z.Y.J., H.S., and T.N.C.. H.F.G. and X.S.X. analyzed the results and edited figures. J.S.X., Y.J.Z., Z.Y.J. and J.Z. performed SCI model and statistical analysis. H.F.G. and L.W. performed cell culture and treatments. H.F.G., J.T.S., J.W. and T.N.C. performed immunoblotting and immunostaining. H.F.G. wrote preliminary draft of the manuscript. S.L.H and H.F. designed experiments and revised the manuscript. All authors approved final version of the manuscript.

\section{Conflict of interest}

The authors declare they have no conflict of interest.

\section{Acknowledge}

Not applicable.

\section{References}

1. Hutson TH, Di Giovanni S (2019) The translational landscape in spinal cord injury: focus on neuroplasticity and regeneration. Nature reviews Neurology 15 (12):732-745. doi:10.1038/s41582-019-0280-3

2. Hu R, Sun H, Zhang Q, Chen J, Wu N, Meng H, Cui G, Hu S, Li F, Lin J, Wan Q, Feng H (2012) G-protein coupled estrogen receptor 1 mediated estrogenic neuroprotection against spinal cord injury. Critical care medicine 40 (12):3230-3237. doi:10.1097/CCM.0b013e3182657560

3. Hu SL, Lu PG, Zhang LJ, Li F, Chen Z, Wu N, Meng H, Lin JK, Feng H (2012) In vivo magnetic resonance imaging tracking of SPIO-labeled human umbilical cord mesenchymal stem cells. Journal of cellular biochemistry 113 (3):1005-1012. doi:10.1002/jcb.23432

4. Hu R, Duan B, Wang D, Yu Y, Li W, Luo H, Lu P, Lin J, Zhu G, Wan Q, Feng H (2011) Role of acid-sensing ion channel 1a in the secondary damage of traumatic spinal cord injury. Annals of surgery 254 (2):353-362. doi:10.1097/SLA.0b013e31822645b4

5. Chen J, Hu R, Ge H, Duanmu W, Li Y, Xue X, Hu S, Feng H (2015) G-protein-coupled receptor 30-mediated antiapoptotic effect of estrogen on spinal motor neurons following injury and its underlying mechanisms. Molecular medicine reports 12 (2):1733-1740. doi:10.3892/mmr.2015.3601

6. Li L, Xiong ZY, Qian ZM, Zhao TZ, Feng H, Hu S, Hu R, Ke Y, Lin J (2014) Complement C5a is detrimental to histological and functional locomotor recovery after spinal cord injury in mice. Neurobiology of disease 66:74-82. doi:10.1016/j.nbd.2014.02.008

7. Yuan J, Liu W, Zhu H, Chen Y, Zhang X, Li L, Chu W, Wen Z, Feng H, Lin J (2017) Curcumin inhibits glial scar formation by suppressing astrocyte-induced inflammation and fibrosis in vitro and in vivo. Brain research 1655:90-103. doi:10.1016/j.brainres.2016.11.002

8. Xia Y, Zhao T, Li J, Li L, Hu R, Hu S, Feng H, Lin J (2008) Antisense vimentin cDNA combined with chondroitinase ABC reduces glial scar and cystic cavity formation following spinal cord injury in rats. Biochemical and biophysical research communications 377 (2):562-566. doi:10.1016/j.bbrc.2008.10.024

9. Xia Y, Yan Y, Xia H, Zhao T, Chu W, Hu S, Feng H, Lin J (2015) Antisense vimentin cDNA combined with chondroitinase ABC promotes axon regeneration and functional recovery following spinal cord injury in rats. Neuroscience letters 590:74-79. doi:10.1016/j.neulet.2015.01.073

10. Hu SL, Luo HS, Li JT, Xia YZ, Li L, Zhang LJ, Meng H, Cui GY, Chen Z, Wu N, Lin JK, Zhu G, Feng H (2010) Functional recovery in acute traumatic spinal cord injury after transplantation of human umbilical cord mesenchymal stem cells. Critical care medicine 38 (11):2181-2189. doi:10.1097/CCM.0b013e3181f17c0e

11. Chu W, Yuan J, Huang L, Xiang X, Zhu H, Chen F, Chen Y, Lin J, Feng H (2015) Valproic Acid Arrests Proliferation but Promotes Neuronal Differentiation of Adult Spinal NSPCs from SCI Rats. Neurochemical research 40 (7):1472-1486. doi:10.1007/s11064-015-1618-x

12. Chen F, Wang H, Xiang X, Yuan J, Chu W, Xue X, Zhu H, Ge H, Zou M, Feng H, Lin J (2014) Curcumin increased the differentiation rate of neurons in neural stem cells via wnt signaling in vitro study. The Journal of surgical research 192 (2):298-304. doi:10.1016/j.jss.2014.06.026

13. Mekhail M, Almazan G, Tabrizian M (2012) Oligodendrocyte-protection and remyelination post-spinal cord injuries: a review. Progress in neurobiology 96 (3):322-339. doi:10.1016/j.pneurobio.2012.01.008 
14. Fehlings MG, Tator $\mathrm{CH}$ (1995) The relationships among the severity of spinal cord injury, residual neurological function, axon counts, and counts of retrogradely labeled neurons after experimental spinal cord injury. Exp Neurol 132 (2):220-228. doi:10.1016/0014-4886(95)90027-6

15. Kakulas BA (1999) A review of the neuropathology of human spinal cord injury with emphasis on special features. The journal of spinal cord medicine 22 (2):119-124. doi:10.1080/10790268.1999.11719557

16. Baumann N, Pham-Dinh D (2001) Biology of oligodendrocyte and myelin in the mammalian central nervous system. Physiological reviews 81 (2):871-927. doi:10.1152/physrev.2001.81.2.871

17. Juurlink BH, Thorburne SK, Hertz L (1998) Peroxide-scavenging deficit underlies oligodendrocyte susceptibility to oxidative stress. Glia 22 (4):371-378. doi:10.1002/(sici)1098-1136(199804)22:4<371::aid-glia6>3.0.c0;2-6

18. Thorburne SK, Juurlink BH (1996) Low glutathione and high iron govern the susceptibility of oligodendroglial precursors to oxidative stress. Journal of neurochemistry 67 (3):1014-1022. doi:10.1046/j.1471-4159.1996.67031014.x

19. Fan BY, Pang YL, Li WX, Zhao CX, Zhang Y, Wang X, Ning GZ, Kong XH, Liu C, Yao X, Feng SQ (2021) Liproxstatin-1 is an effective inhibitor of oligodendrocyte ferroptosis induced by inhibition of glutathione peroxidase 4. Neural regeneration research 16 (3):561-566. doi:10.4103/16735374.293157

20. Shi J, Tang R, Zhou Y, Xian J, Zuo C, Wang L, Wang J, Feng H, Hu S (2020) Attenuation of White Matter Damage Following Deferoxamine Treatment in Rats After Spinal Cord Injury. World neurosurgery 137:e9-e17. doi:10.1016/j.wneu.2019.08.246

21. Dixon SJ, Lemberg KM, Lamprecht MR, Skouta R, Zaitsev EM, Gleason CE, Patel DN, Bauer AJ, Cantley AM, Yang WS, Morrison B, 3rd, Stockwell BR (2012) Ferroptosis: an iron-dependent form of nonapoptotic cell death. Cell 149 (5):1060-1072. doi:10.1016/j.cell.2012.03.042

22. Miotto G, Rossetto M, Di Paolo ML, Orian L, Venerando R, Roveri A, Vučković AM, Bosello Travain V, Zaccarin M, Zennaro L, Maiorino M, Toppo S, Ursini F, Cozza G (2020) Insight into the mechanism of ferroptosis inhibition by ferrostatin-1. Redox biology 28:101328. doi:10.1016/j.redox.2019.101328

23. Abdalkader M, Lampinen R, Kanninen KM, Malm TM, Liddell JR (2018) Targeting Nrf2 to Suppress Ferroptosis and Mitochondrial Dysfunction in Neurodegeneration. Frontiers in neuroscience 12:466. doi:10.3389/fnins.2018.00466

24. Li S, Zhou C, Zhu Y, Chao Z, Sheng Z, Zhang Y, Zhao Y (2020) Ferrostatin-1 alleviates angiotensin II (Ang II)- induced inflammation and ferroptosis in astrocytes. International immunopharmacology 90:107179. doi:10.1016/j.intimp.2020.107179

25. Basso DM, Beattie MS, Bresnahan JC (1996) Graded histological and locomotor outcomes after spinal cord contusion using the NYU weightdrop device versus transection. Experimental neurology 139 (2):244-256. doi:10.1006/exnr.1996.0098

26. Serdar M, Mordelt A, Müser K, Kempe K, Felderhoff-Müser U, Herz J, Bendix I (2019) Detrimental Impact of Energy Drink Compounds on Developing Oligodendrocytes and Neurons. Cells 8 (11). doi:10.3390/cells8111381

27. Han S, Tang Q, Chen R, Li Y, Shu J, Zhang X (2017) Hepatic iron overload is associated with hepatocyte apoptosis during Clonorchis sinensis infection. BMC infectious diseases 17 (1):531. doi:10.1186/s12879-017-2630-3

28. Jiang X, Zhang J, Kou B, Zhang C, Zhong J, Fang X, Huang X, Zhang X, Xie F, Hu Q, Ge H, Yu A (2020) Ambroxol Improves Neuronal Survival and Reduces White Matter Damage through Suppressing Endoplasmic Reticulum Stress in Microglia after Intracerebral Hemorrhage. BioMed research international 2020:8131286. doi:10.1155/2020/8131286

29. Muhoberac BB, Vidal R (2019) Iron, Ferritin, Hereditary Ferritinopathy, and Neurodegeneration. Frontiers in neuroscience $13: 1195$. doi:10.3389/fnins.2019.01195

30. Bin JM, Harris SN, Kennedy TE (2016) The oligodendrocyte-specific antibody 'CC1' binds Quaking 7. Journal of neurochemistry 139 (2):181186. doi: $10.1111 /$ jnc. 13745

31. Xie BS, Wang YQ, Lin Y, Mao Q, Feng JF, Gao GY, Jiang JY (2019) Inhibition of ferroptosis attenuates tissue damage and improves long-term outcomes after traumatic brain injury in mice. CNS neuroscience \& therapeutics 25 (4):465-475. doi:10.1111/cns.13069

32. Ling X, Liu D (2007) Temporal and spatial profiles of cell loss after spinal cord injury: Reduction by a metalloporphyrin. Journal of neuroscience research 85 (10):2175-2185. doi:10.1002/jnr.21362

33. Ek CJ, Habgood MD, Callaway JK, Dennis R, Dziegielewska KM, Johansson PA, Potter A, Wheaton B, Saunders NR (2010) Spatio-temporal progression of grey and white matter damage following contusion injury in rat spinal cord. PloS one 5 (8):e12021. doi:10.1371/journal.pone.0012021

34. Lachaier E, Louandre C, Godin C, Saidak Z, Baert M, Diouf M, Chauffert B, Galmiche A (2014) Sorafenib induces ferroptosis in human cancer cell lines originating from different solid tumors. Anticancer research 34 (11):6417-6422

35. Stockwell BR, Friedmann Angeli JP, Bayir H, Bush Al, Conrad M, Dixon SJ, Fulda S, Gascón S, Hatzios SK, Kagan VE, Noel K, Jiang X, Linkermann A, Murphy ME, Overholtzer M, Oyagi A, Pagnussat GC, Park J, Ran Q, Rosenfeld CS, Salnikow K, Tang D, Torti FM, Torti SV, Toyokuni S, Woerpel KA, Zhang DD (2017) Ferroptosis: A Regulated Cell Death Nexus Linking Metabolism, Redox Biology, and Disease. Cell 171 (2):273-285. doi:10.1016/j.cell.2017.09.021

36. Magtanong L, Dixon SJ (2018) Ferroptosis and Brain Injury. Developmental neuroscience 40 (5-6):382-395. doi:10.1159/000496922 
37. Weiland A, Wang Y, Wu W, Lan X, Han X, Li Q, Wang J (2019) Ferroptosis and Its Role in Diverse Brain Diseases. Molecular neurobiology 56 (7):4880-4893. doi:10.1007/s12035-018-1403-3

38. Datta A, Sarmah D, Mounica L, Kaur H, Kesharwani R, Verma G, Veeresh P, Kotian V, Kalia K, Borah A, Wang X, Dave KR, Yavagal DR, Bhattacharya P (2020) Cell Death Pathways in Ischemic Stroke and Targeted Pharmacotherapy. Translational stroke research 11 (6):11851202. doi:10.1007/s12975-020-00806-z

39. Hendriks KDW, Joschko CP, Hoogstra-Berends F, Heegsma J, Faber KN, Henning RH (2020) Hibernator-Derived Cells Show Superior Protection and Survival in Hypothermia Compared to Non-Hibernator Cells. International journal of molecular sciences 21 (5). doi:10.3390/ijms21051864

40. Li YZ, Zhou XL, Huo BQ, Chen DZ, Liu ZH, Sheng XH (2019) Reactions of the Lipid Hydroperoxides With Aminic Antioxidants: The Influence of Stereoelectronic and Resonance Effects on Hydrogen Atom Transfer. Frontiers in chemistry 7:850. doi:10.3389/fchem.2019.00850

41. Song J, Liu T, Yin Y, Zhao W, Lin Z, Yin Y, Lu D, You F (2021) The deubiquitinase OTUD1 enhances iron transport and potentiates host antitumor immunity. EMBO reports:e51162. doi:10.15252/embr.202051162

42. Yao X, Zhang Y, Hao J, Duan HQ, Zhao CX, Sun C, Li B, Fan BY, Wang X, Li WX, Fu XH, Hu Y, Liu C, Kong XH, Feng SQ (2019) Deferoxamine promotes recovery of traumatic spinal cord injury by inhibiting ferroptosis. Neural regeneration research 14 (3):532-541. doi:10.4103/16735374.245480

43. Louandre C, Ezzoukhry Z, Godin C, Barbare JC, Mazière JC, Chauffert B, Galmiche A (2013) Iron-dependent cell death of hepatocellular carcinoma cells exposed to sorafenib. International journal of cancer 133 (7):1732-1742. doi:10.1002/ijc.28159

44. Zhang Y, Fan BY, Pang YL, Shen WY, Wang X, Zhao CX, Li WX, Liu C, Kong XH, Ning GZ, Feng SQ, Yao X (2020) Neuroprotective effect of deferoxamine on erastininduced ferroptosis in primary cortical neurons. Neural regeneration research 15 (8):1539-1545. doi:10.4103/16735374.274344

\section{Figures}

A

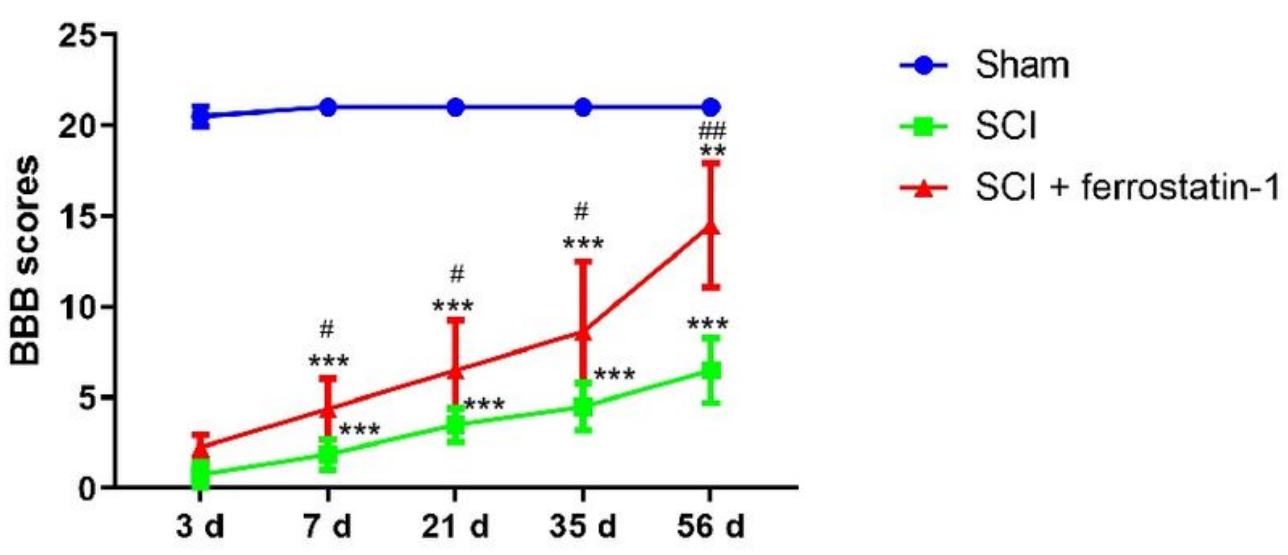

C

Sham

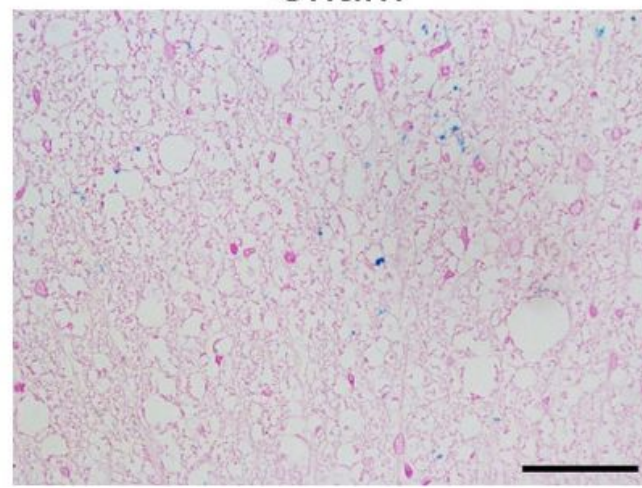

$\mathrm{SCl}$

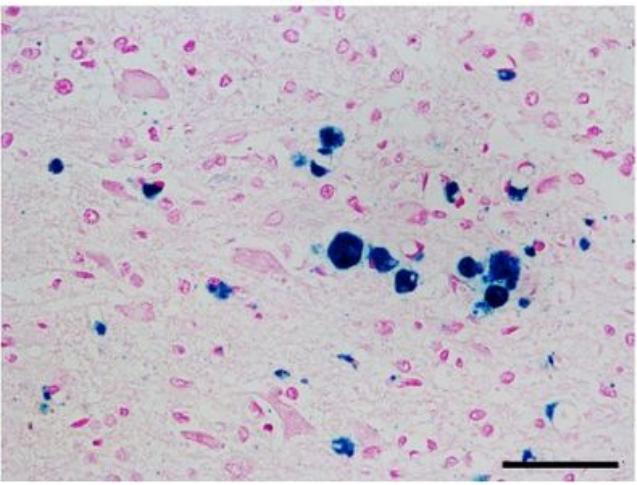

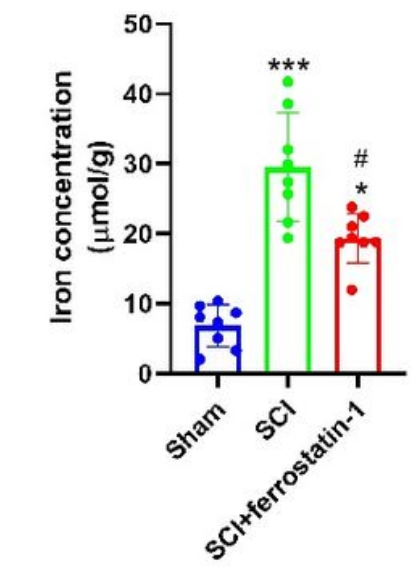

B

Figure 1 
Ferrostatin-1 promotes functional recovery through reducing iron deposition in rats after spinal cord injury (SCl). (A) Summarized data showing the post-injury motor behavior using Basso, Beattie and Bresnahan (BBB) score in different groups on day $3,7,21,35$ and 56 post-SCl. ***P<0.01 vs.

Sham; ${ }^{\star *} \mathrm{P}<0.01$ vs. Sham; $\# \mathrm{P}<0.05$, \#\#P<0.01 vs. $\mathrm{SCl}$. (B) The iron concentration in the epicenter of injured spinal cord on day 7 in different groups.

(C) Perl's Prussian blue staining images showing the iron accumulation in the epicenter of injured spinal cord on day 7 in different groups. Scale bar: $50 \mu \mathrm{m}$.

A
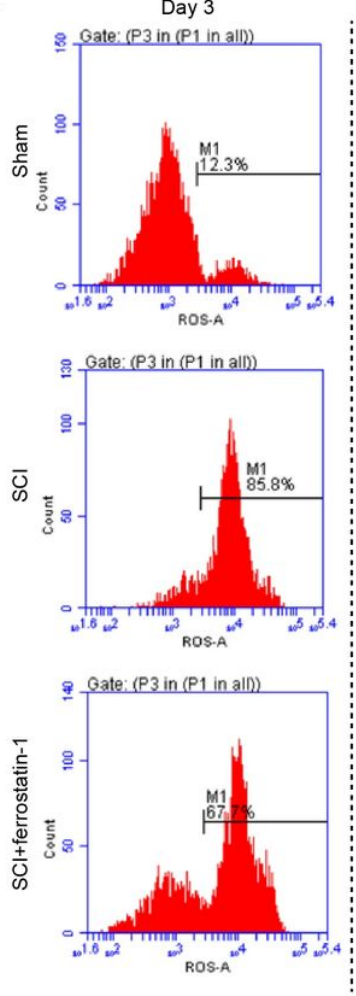

Day 7
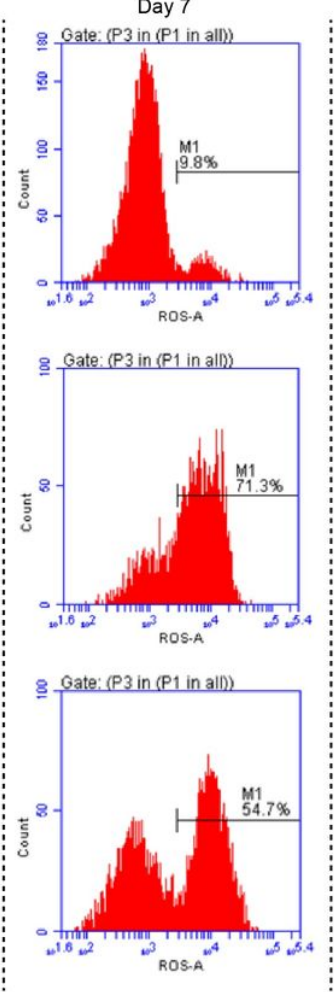

Day 21
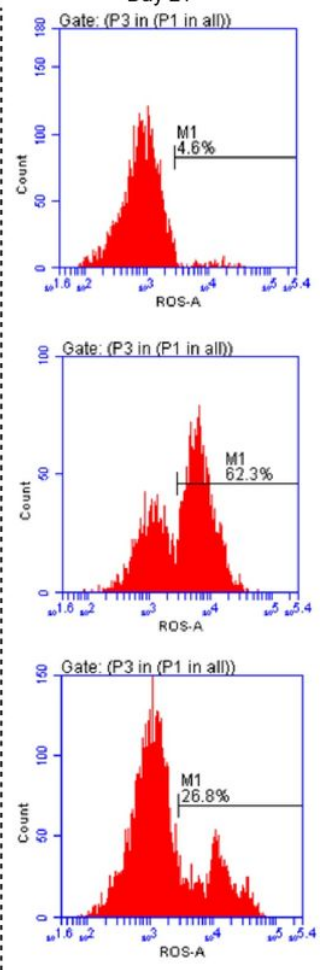

B

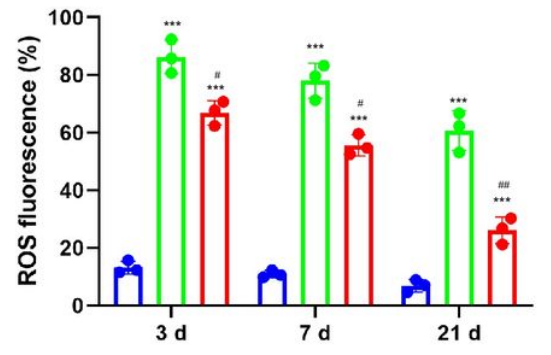

- Sham

- $\mathrm{SCl}$

- $\mathrm{SCl}+$ ferrostatin-1

Figure 2

Ferrostatin-1 reduces reactive oxygen species (ROS) accumulation in the epicenter post-SCl. (A) Representational images indicating the ROS accumulation using Flow Cytometry. (B) Bargraph summarizing the percentage of ROS fluorescence from (A). ${ }^{* *} \mathrm{P}<0.01$ vs. Sham; \#P<0.05, \#\#P<0.01 vs. SCl. 


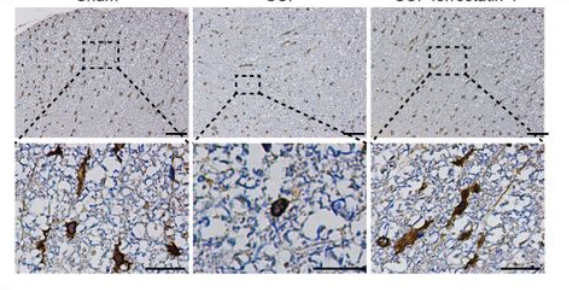

C

D

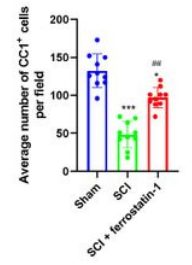

E
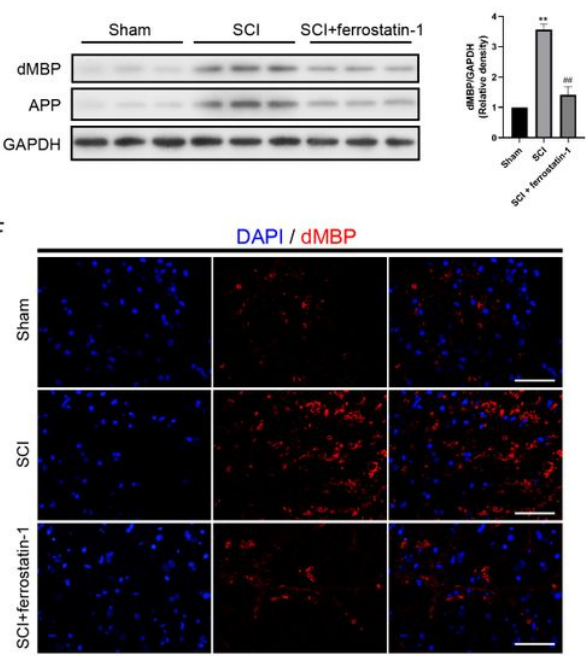

G

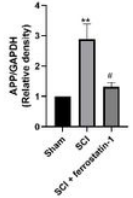

H
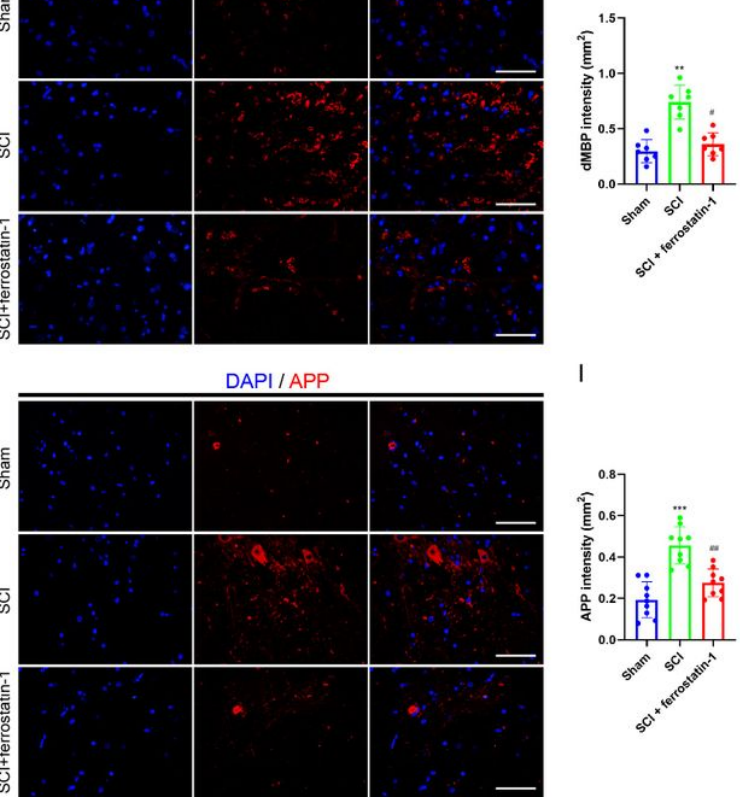

I

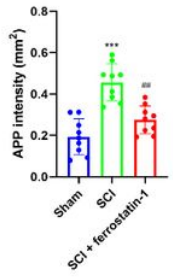

Figure 3

Ferrostatin-1 decreases demyelination on day 7 post-SCI. (A) Representative immunohistochemistry images of CC1 in the epicenter of injured spinal cord on day 7 in different groups. Scale bar: $50 \mu \mathrm{m} ; 10 \mu \mathrm{m}$ for enlarged images. (B) Barchart indicating the average number of CC1+ cells per field $(200 \times) .{ }^{* *} \mathrm{P}<0.01,{ }^{*} \mathrm{P}<0.05$ vs. Sham; \#\#P<0.01 vs. SCI. (C) Immunoblot bands representing the expression level of dMBP and APP in various groups. GAPDH was served as an internal control. (D, E) Semi-quantitative analysis of the expression of dMBP (D) and APP (E) from (C). **P<0.01 vs. Sham; \#P<0.05, \#\#P<0.01 vs. SCl. (F) Immunostaining images delineating the expression of dMBP in each group. Nuclei were counterstained with DAPI. Scale bar: $20 \mu \mathrm{m}$. (G) Semi-quantitative data showing the optic density of dMBP in each group. ${ }^{*} \mathrm{P}<0.01$ vs. Sham; \#P<0.05 vs. SCI. (H) Immunostaining images demonstrating the expression of APP in each group. Scale bar: $20 \mu \mathrm{m}$. (H) Semi-quantitative bargraph indicating the optic density of APP in each group. ***P<0.001 vs. Sham; \#\#P<0.01 vs. SCl. 
A
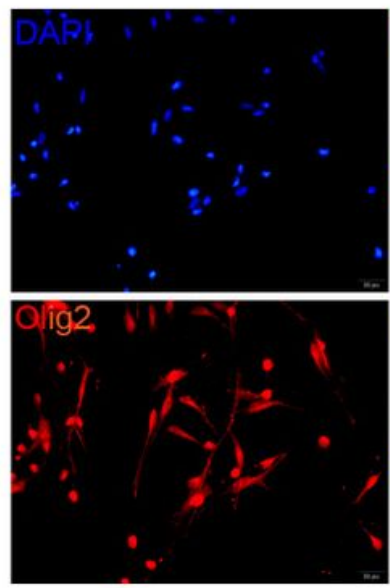

C

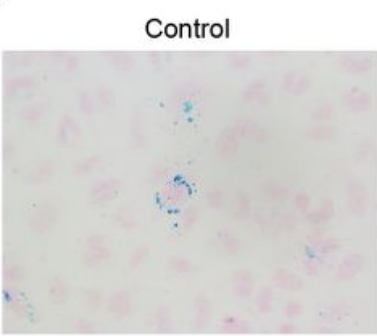

$\mathrm{E}$

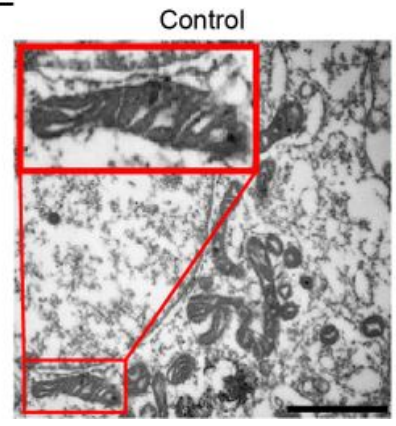

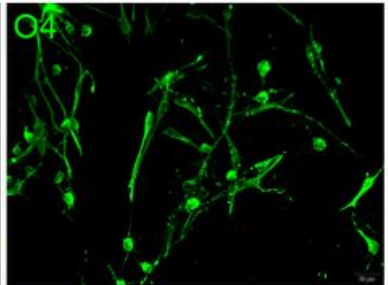

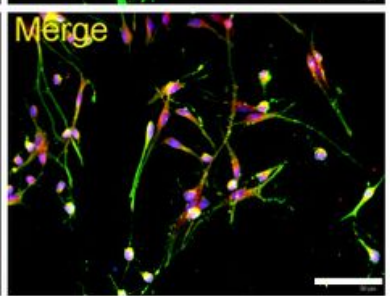

B
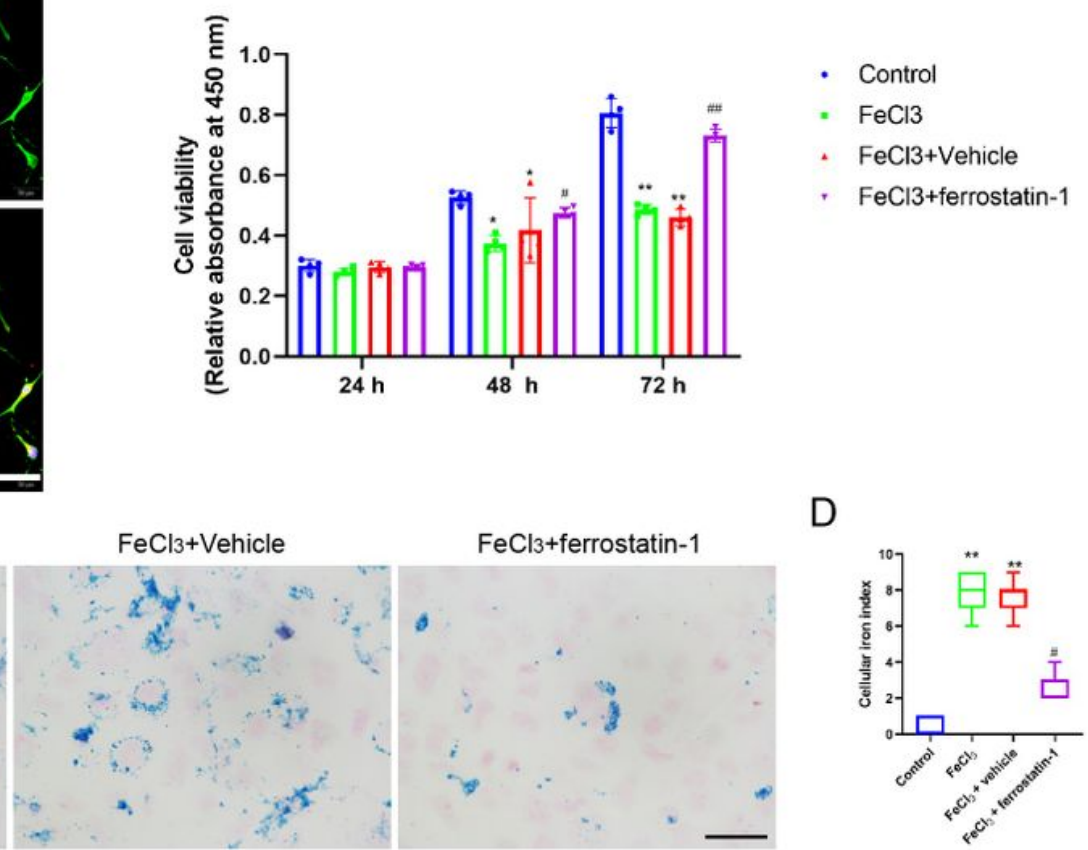

$\mathrm{F}$
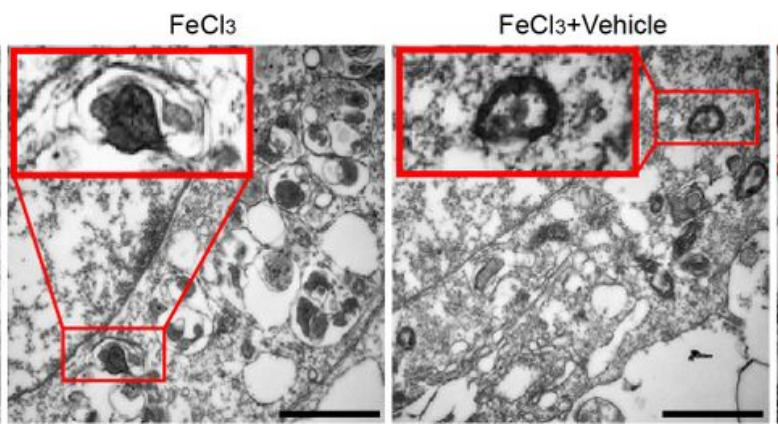

$\mathrm{FeCl}_{3}+$ ferrostatin-1
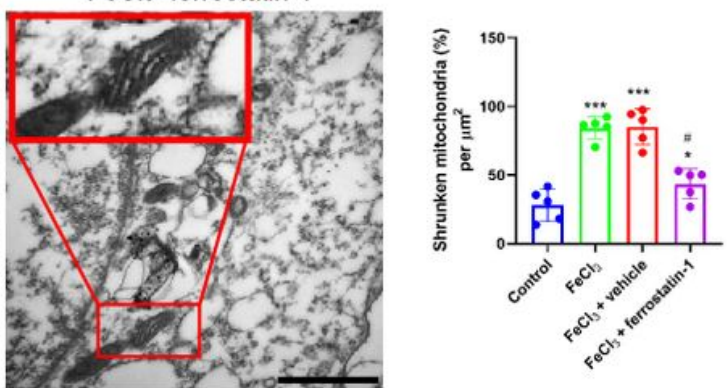

Figure 4

Ferrostatin-1 reduces OPCs injury induced by iron overload through reducing ferroptosis in vitro. (A) Representative immunostaining images of 04 (green) and Olig2 (red) in primary OPCs. Nuclei were counterstained with DAPI. Scale bar: $20 \mu \mathrm{m}$. (B) Barchart showing cell viability determined by CCK8 in each group at different time points. ${ }^{*} \mathrm{P}<0.05,{ }^{*} \mathrm{P}<0.01$ vs. Control; $\# \mathrm{P}<0.05, \# \# \mathrm{P}<0.01$ vs. $\mathrm{FeCl} 3$ or $\mathrm{FeCl} 3+$ Vehicle. (C) Perl's Prussian blue staining images showing the iron accumulation in primary oligodendrocytes at $48 \mathrm{~h}$ in different groups. Scale bar: $50 \mu \mathrm{m}$. (D) Summarized data indicating cellular iron index from (C). ${ }^{*} \mathrm{P}<0.01$ vs. Control; $\# \mathrm{P}<0.05$ vs. $\mathrm{FeCl} 3$ or $\mathrm{FeCl} 3+$ Vehicle. (E) Transmission electron microscopy of primary

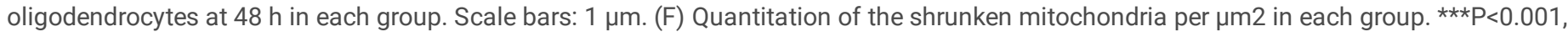
${ }^{*} \mathrm{P}<0.05$ vs. Control; $\# \mathrm{P}<0.05$ vs. $\mathrm{FeCl} 3$ or $\mathrm{FeCl} 3+$ Vehicle. 
A

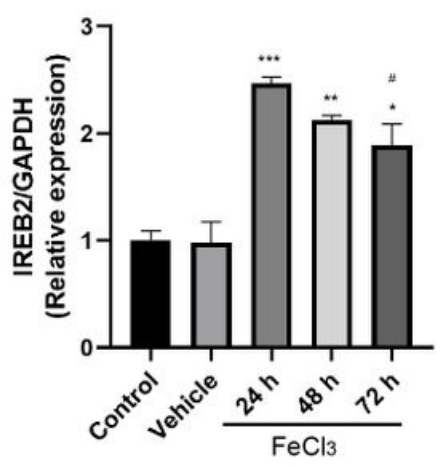

B

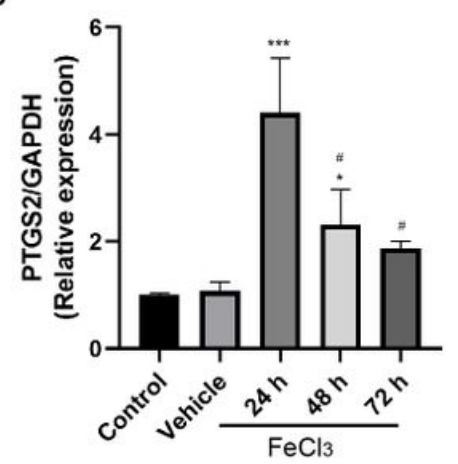

C

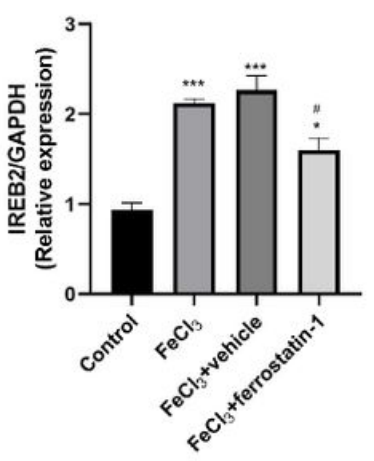

F

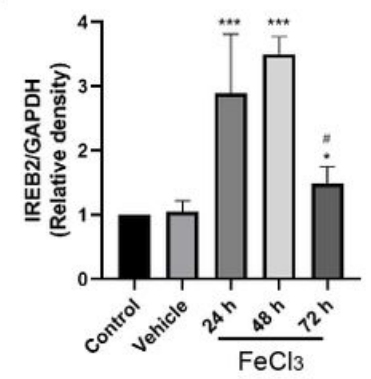

I

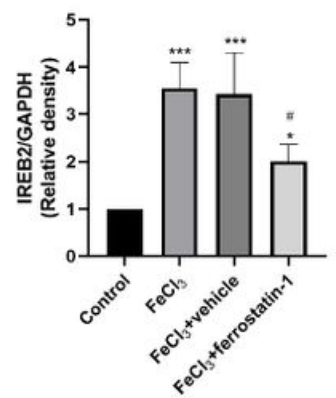

D

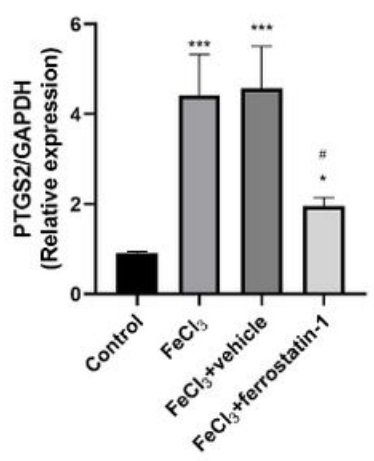

G

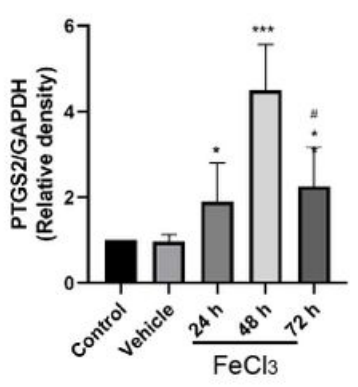

J

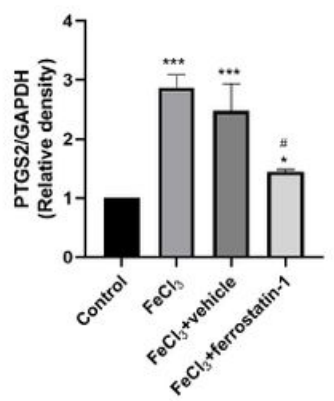

Figure 5

Ferrostatin-1 alleviates OPCs ferroptosis resulting from iron overload through decreasing IREB2 expression. (A, B) IREB2 (A) and PTGS2 (B) mRNA expression evaluated by RT-qPCR in each group at different time points. ${ }^{* *} P<0.001,{ }^{*} \mathrm{P}<0.01,{ }^{*} \mathrm{P}<0.05$ vs. Control or Vehicle; $\# \mathrm{P}<0.05$ vs. FeCl3 at $24 \mathrm{~h}$ or $48 \mathrm{~h}$. (C, D) IREB2 (C) and PTGS2 (D) mRNA expression evaluated by RT-qPCR with ferrostatin-1 administration at $48 \mathrm{~h}$ in each group. ${ }^{*} * * \mathrm{P}<0.001,{ }^{*} \mathrm{P}<0.05$ vs. Control; \#P<0.05 vs. FeCl3 or FeCl3 + Vehicle. (E) Immunoblot bands representing the expression level of IREB2 and $\mathrm{PTGS} 2$ in each group at different time points. GAPDH was served as an internal control. (F, G) Semi-quantitative analysis of the expression of IREB2 (F) and PTGS2 (G) from (E). ${ }^{\star * *} \mathrm{P}<0.001,{ }^{*} \mathrm{P}<0.05$ vs. Control or Vehicle; $\# \mathrm{P}<0.05$ vs. FeCl3 at $24 \mathrm{~h}$ or $48 \mathrm{~h}$. $(\mathrm{H})$ Immunoblot bands indicating the expression level of IREB2 and PTGS2 at $48 \mathrm{~h}$ in each group. GAPDH was served as an internal control. (I, J) Semi-quantitative analysis of the expression of IREB2 (I) and PTGS2 (J) from (H). ${ }^{* \star *} \mathrm{P}<0.001$, ${ }^{*} \mathrm{P}<0.05$ vs. Control; $\# \mathrm{P}<0.05$ vs. FeCl3 or FeCl3 $+\mathrm{Vehicle.}$ 